\title{
Una historia crítica del concepto de experiencia de la epistemología feminista
}

\author{
A CRITICAL HISTORY OF THE CONCEPT OF EXPERIENCE IN FEMINIST EPISTEMOLOGY
}

Catalina Trebisacce (catalinaptrebisacce@gmail.com) Consejo Nacional de Investigaciones Científicas y Tecnológicas (Buenos Aires, Argentina) ORCID: 0000-0002-6601-8858

\begin{abstract}
This article analyzes the innovations produced by the concept of experience, introduced from the feminist theory during the eighties. The experience was an epistemic invention to give account of what used to result exceeding, subsidiary, or invisible to the science legitimated as such. This theoreticalmethodological tool led to redefinitions around the sense of objectivity and pointed out the political condition of a perspective that was declared as neutral. This work tries to throw some light over the critical strength that this epistemic tool had during those years, for which it chooses the historical perspective. At the same time, this article advances towards a critical analysis around certain modelling of the tool. In this sense, the article aims to sharpen the epistemic surveillance and to review the commitments that this has been acquiring with the institutionalization of the feminist perspective during the last decades.
\end{abstract}

Key words: feminist epistemology, experience, history, institutionalization, gender study.

\section{Resumen}

El presente artículo analiza las innovaciones que produjo en el campo del conocimiento la herramienta de la experiencia, introducida desde la teoría feminista en los años ochenta. La experiencia fue una invención epistémica que tuvo como objetivo dar cuenta de aquello que resultaba excedente, subsidiario o invisible a la ciencia legitimada como tal. Este instrumental teórico-metodológico conllevó redefiniciones en torno al sentido de la objetividad y señaló la condición política de una perspectiva declarada neutral. El presente trabajo apunta a alumbrar la potencia crítica que dicha herramienta epistémica tuvo en aquellos años, para lo cual elige la perspectiva histórica. Asimismo, el artículo avanza en un análisis crítico en torno a ciertas modelizaciones contemporáneas de dicha herramienta. En este sentido, el artículo aspira a agudizar la vigilancia epistémica y a revisar los compromisos que ha ido adquiriendo con la institucionalización de la perspectiva feminista de las últimas décadas.

Palabras clave: epistemología feminista, experiencia, historia, institucionalización, estudios de género.

\section{Introducción}

El presente artículo se propone un análisis histórico en torno a la incorporación de los estudios de género en los centros legitimados de producción de conocimiento que se produce en la década del ochenta. Con anterioridad a esta década algunas expresiones del movimiento feminista norteamericano habían llegado a convocar a las mujeres a hacer arder sus títulos universitarios por considerarlos resultado de un conocimiento androcéntrico y opresor. Pero en los ochenta se presentan otras estrategias cuando algunas 
mujeres comenzaron a explorar la posibilidad de producir conocimiento científico informado en una perspectiva feminista que pudiera poner en cuestionamiento los sesgos del conocimiento autodeclarado neutral. Sobre estas otras estrategias versará este artículo y sobre las implicancias epistémicas y políticas que ellas han introducido en la producción de conocimiento en el campo de las ciencias sociales y humanísticas.

Concretamente, el artículo se ocupa de analizar las innovaciones que produjo en el campo del conocimiento la herramienta epistémica de la experiencia introducida desde la teoría feminista en los años ochenta. Se despliega para ello, en primer lugar, un análisis histórico que permite comprender las razones coyunturales y políticas del surgimiento de los estudios de género, en general, y de aquella noción, en particular. En segundo lugar, se exponen y se analizan las torsiones y las rupturas que la experiencia, en tanto que instrumento para la producción de conocimiento, introdujo a los dispositivos por entonces disponibles en el campo del saber. Finalmente, se señalan y se ponderan algunos riesgos ligados la institucionalización de los estudios de género que podrían estar produciendo un vaciamiento de su potencial crítico, epistémico y político de la noción en cuestión.

\section{Yeguas de Troya o cómo tomar la academia por asalto}

El feminismo de los años 70 conmovió a buena parte de la sociedad occidental y llegó a tocar las puertas de instancias de poder político internacional como la Organización de las Naciones Unidas, que en 1975 declaró a aquel año como el Año Internacional de la Mujer y el inicio de la Década de la Mujer, con el fin de promover la implementación de medidas orientadas a generar una mayor equidad de género en distintos aspectos de la vida de las mujeres, incluidos aquellos en relación con la producción de conocimiento. Se aconsejó a cada Estado Parte no solo ampliar el número de mujeres en sus organismos, sino también producir conocimiento específico en torno a la situación y calidad de vida de las mujeres en cada lugar. Sin bien la injerencia real de estas políticas internacionales no deben ser sobreestimadas, sí es necesario advertir que en los años siguientes las feministas se encontraron con este aliado particular y con la posibilidad de acceso a distintos lugares de poder, entre ellos los centros universitarios. Advinieron entonces preocupaciones respecto de la manera de ocupar esos espacios ganados. Los contextos particulares depararon situaciones distintas para cada punto geopolítico, pero en lo que refiere al ingreso de la perspectiva crítica del feminismo en los más o menos higiénicos laboratorios de producción de conocimiento, como son las universidades, las características del ingreso han sido similares. Se especuló con todo tipo de estrategias para el pasaje. Y aunque no fue una metáfora explicitada, la idea de devenir yeguas de troya organizó aquella experiencia. Ingresar al templo del saber con atuendos para la ocasión y una vez allí adentro dar todas las peleas. Pero pronto -o simultáneamente- emergía otras inquietudes: ¿puede ser feminista la construcción del conocimiento? Fue la pregunta recurrente cuyo espíritu fue eternizado en uno de los textos más viejos y revisitados de epistemología y metodología escrito desde el feminismo. Me refiero al artículo de Sandra Harding publicado a mediados de los ochenta bajo el título “¿Existe un método feminista?"

Menciono este texto de Harding por asociación sintagmática, pero corresponde decir que la pregunta por la epistemología feminista es una inquietud que gravitó la década del 80, escenario de la aparición de una voluminosa cantidad de textos que intentaron abordar el complicado cruce entre la producción de conocimiento científico y feminismo. Fue esta década, y no otra, porque coincide con el ingreso de la perspectiva feminista a los centros legitimados de producción de conocimiento, es decir, las universidades. 
Hasta ese momento el movimiento feminista, huérfano de instituciones del saber, huérfano de tradiciones teóricas, había producido con cierta precariedad, pero con el encanto de la producción artesanal y experimental, un conocimiento que hoy denominaríamos "informal", o "pagano" para el sacro conocimiento académico. Especialmente en los años 70, en el norte, pero también aquí en el sur, las feministas compartieron en grupos de concienciación sus propias experiencias con el objetivo de analizar malestares estructurales que no eran contemplados ni por saberes disponibles ni por programas de políticas emancipatorias. Traficaron trabajosamente ideas de feministas de otros lugares. Produjeron fanzines, artículos, revistas y libros. Improvisaron una lengua teórica con la que denunciaron un sistema de opresión invisibilizado, no solo por la sociedad en su conjunto, sino también por las agrupaciones políticas de casi todo el arco de la izquierda revolucionaria. Escribieron Mística de la femineidad de Betty Friedam; Política Sexual de Kate Millet; Escupamos sobre Hegel de Carla Lonzi, por nombrar algunos de los textos fundamentales del feminismo de entonces. Estos textos escritos en su mayoría en la periferia de los centros universitarios, compuestos en una "lengua tartamuda", como le gusta decir a Donna Haraway (1995:336), porque puede dar explicaciones tan solo de algo parcialmente comprendido, fueron sin embargo de una potencia crítica destacable y alcanzaron una importante repercusión.

Esta lengua teórica pero artesanal, finalmente tartamuda, interpeló a toda una generación de mujeres, algunas de las cuales ingresaron en los centros universitarios en la década del 80 y comenzaron la tarea de creación de institutos y departamentos que pudieran ser espacios para el desarrollo de estudios críticos con perspectiva feminista. Es con este ingreso (que fue de algún modo una toma por asalto) que se iniciaron las reflexiones específicas en torno a una posible (o imposible) epistemología feminista. No es que con anterioridad no hubiera habido estudios en los que se considerara la situación de la población femenina. Por ejemplo, desde la antropología, ya en la década del 20 Margaret Mead se había ocupado del estudio de los roles sexuales entre los samoanos. Preocupación que continuó con distintos estudios hasta bien entrados los años 40. Y no fue la única. En la década del 70 algunas antropólogas, como Sherry Ortner o Michelle Rosaldo, se interesaron en el estudio específico de la situación de las mujeres en distintas sociedades. Incluso, podríamos mencionar el famosísimo trabajo de Gayle Rubín El tráfico de mujeres: Notas sobre la "economía política" del sexo de 1975, luego recuperado casi como escrito fundante del campo de los estudios de género. Ahora bien, ninguno de estos trabajos, ni tantos otros que no mencioné, dieron lugar a reflexiones epistémicas específicas. Ellas arribaron recién en los 80 de la mano del ingreso de mujeres que traían consigo la lengua del feminismo de las calles y presionaban con la creación de centros de estudio especializados. Fue, entonces, a partir del encuentro de una perspectiva que se reconoce política y situada, como lo es el feminismo, con un dispositivo de producción de conocimiento que se declara (se miente) neutral y universal, que se abrió la necesidad de un debate epistémico específico.

El ingreso del feminismo a la academia supuso una traducción ( $y$, por definición, una traición) de la lengua tartamuda al lenguaje científico. Lo que, en otras palabras, fue (y es) una negociación (a veces mejor llevada, a veces peor) en torno a compromisos epistémicos y políticos en la producción de conocimiento científico. Me interesa señalar este momento, el de los años 80 , el de la traducción, el del ingreso al templo del saber de la lengua combativa, para no perder de vista que el conocimiento que se produce desde entonces en la academia es un tipo específico de conocimiento entre otros, no solo posibles, sino ya existentes. $Y$, de este modo, recordar que de lo que vamos a discurrir aquí tiene una historia y una prehistoria, ambas plagadas de conflictos y de tensiones, pero también de estrategias y de decisiones, todas ellas políticas y epistémicas. 


\section{Una revuelta epistémica o la experiencia insurrecta agrietando los muros del saber}

Hubo quienes pensaron, o quisieron pensar, que los estudios de género llegaban a la academia con el objetivo de llenar los vacíos que el conocimiento científico aún no había conseguido completar. Que se trataba de la aparición de una perspectiva que faltaba o de la creación de un nuevo nicho de investigación especializada. Operó entonces la ficción de progresividad de la ciencia. Pero lo cierto es que no hay faltas o vacíos en el conocimiento científico, hay, en todo caso, silenciamientos. Y esto lo saben los estudios de género que, en tanto que saberes sometidos, diría Foucault "conservan la memoria de los enfrentamientos" (1992:137), que no es otra cosa que la memoria del destierro. Los estudios de género no ingresaron prolijamente a la academia sino a los empujones. Nacieron, como sostiene Haraway, con la inocencia epistémica perdida. Conocieron desde un inicio las relaciones de poder que se cruzan, producen y sostienen al conocimiento científico. Las investigaciones con perspectiva feminista se supieron siempre hijas no deseadas de dicho conocimiento, y comprendieron también que su exclusión había sido fundamental para la construcción de la ciencia tal y como existía hasta entonces.

En este sentido, los estudios de género entendieron que el método científico operaba al modo no del instrumento, sino de la máquina. Es decir, no había manera de emplear los mismos caminos (fabulando un uso instrumental) para dar lugar a una nueva perspectiva. El método científico era máquina creadora de productos determinados que suponían, entre otras cosas, la exclusión de la perspectiva feminista. Hubo entonces que subvertir el método, ir contra el método. Escarbar a contrasentido de lo esperado y gestar nuevos marcos interpretativos que no solo no coincidirían con los existentes, sino que los pondrían en cuestión allí donde se sentían ellos mismos más seguros. Así es que, al experimento, fetiche de la construcción del conocimiento de la ciencia neutral, en el que las variables están ponderadas, medidas y controladas en un higiénico laboratorio teórico, aséptico incluso del/de la propio/a investigador/a, los estudios de género opusieron la experiencia (invención contaminada que se sabe, siempre y por definición, inaprensible) como el camino honrosamente precario para producir una perspectiva-otra que resultara inesperada para la ciencia imperante.

Cabe señalar que la gran revuelta contra el método (para seguir jugando con la expresión de Paul Feyeraben) antecede y excede a la irrupción de los estudios de género. Podríamos retrotraerla, si quisiésemos, a la revolución que agitó Thomas Kuhn con La estructura de las revoluciones científica en el campo de las ciencias duras, que produjo heridas irreversibles al espíritu soberbio y desenfadadamente entusiasta de la ciencia progresiva, con inmensurable impacto en todos los campos del conocimiento científico. Pero los sentidos en los que se produjo la crítica epistémica feminista son singulares y su propuesta por la experiencia también lo es.

La experiencia fue convocada para que pudiera hablar de aquello para lo cual la ciencia neutral no tenía ni palabras ni protocolo de experimentación. Y esta ausencia de un lenguaje y de un método disponible para visibilizar estas realidades-otras, fue bien interpretada (y denunciada) como la existencia y el despliegue de un poder. Un poder de mordaza. Poder que desprecia, deslegitima, subestima y, finalmente, no ve sujetos subalternos; ni sus historias, ni sus condiciones específicas de existencia. Dorothy Smith es una de las teóricas feministas que en esta década postulaba la necesidad de la experiencia, explicando que: "no existía un discurso desarrollado en el que las experiencias que designábamos originariamente como experiencias cotidianas pudieran ser traducidas a un lenguaje público y se convirtieran en políticas, en el lenguaje característico del movimiento de las mujeres [....] Hablar de nuestras experiencias fue una forma de descubrimiento" (2012:7). 
No se trató de la experiencia vuelta simplemente objeto de estudio, como lo tomarían algunos de los contemporáneos estudios de la subjetividad humana (Cornejo, Albornoz y Palacios 2016). La experiencia fue citada a dar testimonio de formas de dominio y de opresión productoras de sujetos subalternos. Sujetos dos veces subalternizados a efecto del poder de forclusión desplegado por el propio conocimiento científico: "[la herramienta de la experiencia] se diseñó para crear una alternativa al sujeto deshumanizado del conocimiento del discurso científico social establecido. Este último se ajusta y está incorporado a lo que he dado en llamar 'relaciones de dominación', ese extraordinario y aún habitual complejo de relaciones que están textualmente mediadas, las que nos conectan a través del tiempo y del espacio y organizan nuestras vidas cotidianas" (Smith 2012:10).

La experiencia fue invención resistente, estrategia de disputa, funcionó al modo de un indicador químico, diría Foucault, para visibilizar no sustancias incoloras esta vez, sino poderes naturalizados que nos producen subalternos, al tiempo que nos niegan. Fue, también, y por ello, fuerza corrosiva de esos poderes.

En este sentido, la experiencia es la que permitió la construcción de un conocimiento que rehúye y rechaza las ilusiones de la omnipotencia del conocimiento neutral y des-encarnado que quiere combatir. El conocimiento producido desde la experiencia es siempre conocimiento parcial, y por ello situado. Y el conocimiento situado es el único que comporta la responsabilidad ética de su construcción. Entonces, la construcción de un conocimiento que parta de la experiencia no solo es la posibilidad de la construcción de una herramienta metodológica que permita visualizar estados de cosas inéditos para la ciencia, sino que es una herramienta que abraza simultáneamente compromisos éticos. En este sentido, sostiene Donna Haraway: "lucho a favor de políticas y de epistemologías de la localización, del posicionamiento y de la situación, en las que la parcialidad y no la universalidad es la condición para que sean oídas las pretensiones de lograr un conocimiento racional. Se trata de pretensiones sobre las vidas de la gente, de la visión desde un cuerpo, siempre un cuerpo complejo, contradictorio, estructurante y estructurado, contra la visión desde arriba, desde ninguna parte, desde la simpleza" (1995:335).

El camino de la experiencia es, por tanto, no solo el "sin-remedio" ante la ausencia de la lengua disponible en la ciencia, sino un horizonte político para la construcción de un conocimiento científico. La experiencia, como herramienta epistémica y política, fue el gran aporte, y la gran piedra en el zapato, introducida por los estudios de género al saber académico.

\section{III. ¿La institucionalización de la experiencia? o la victoria que puede ser derrota}

En esta tercera y última sección quisiera compartir algunas inquietudes que en ocasiones me advienen y que entiendo pueden estar ligadas a las ya más de tres décadas de institucionalización de la perspectiva feminista en la academia. Institucionalización sobre la que comienza a ser menester reflexionar suspendiendo por un momento la -aún muy razonable- denuncia sobre la marginación de estos estudios en el campo del saber. Se vuelve necesidad también poder analizar los efectos que encontramos tras el hecho de haber alcanzado un lugar más o menos legitimado, tanto ante colegas como ante la sociedad. El feminismo no debería caer en la tentación de considerarse inmune o a salvo de posibles clausuras analíticas, que ya sabemos se producen casi a efecto obligado de participar de las instituciones legitimadas en la producción de conocimiento.

Considerando entonces esta actualidad de los estudios de género, quisiera realizar algunas observaciones críticas en torno a dos procesos en curso que se despliegan produciendo redefiniciones sobre la noción de 
experiencia en un sentido que termina endureciendo la herramienta y poniendo en riesgo su espíritu disidente y desestabilizador.

Voy a referirme en primer lugar a lo que entiendo es una trasmutación del sentido y del valor gnoseológico y político de la propia noción de experiencia. Algunas teóricas feministas habían advertido ya sobre la posibilidad de un romanticismo respecto de la consideración de la experiencia de las mujeres (Haraway 1995) o sobre la posible esencialización de la perspectiva del punto de vista privilegiado (Scott 1992). Yo quisiera profundizar en torno a estas advertencias y analizar cuáles son sus implicancias epistémicas y políticas, que se enlazan luego con el segundo problema que quisiera desarrollar. Pues en segundo lugar voy a intentar pensar cómo esta transformación de la noción se liga con la progresiva instalación de una interpretación privilegiada de las experiencias de las mujeres.

\section{i. La trasmutación del valor gnoseológico y político de la experiencia o el retorno del individuo}

En la tarea de sostener una vigilancia epistemológica, como desearían Bourdieu, Chamboredon y Passeron (2008), sobre la producción del campo de los estudios de género, se vuelve necesario llamar la atención en torno al hecho de que algunos estudios recientes del área han comenzado a servirse de la experiencia en un sentido que parece desandar algunas de las potencias de dicha noción. Más que como un camino para develar el funcionamiento de un poder o varios poderes, y así donar información epistémica y política sobre las condiciones sociales de existencia, estos estudios han tendido a concebir a los relatos biográficos como relatos que comporta un valor en sí mismo.

Acontece, entonces, una gran confusión que en realidad es la cuña de una perspectiva liberal que se camufla y se cuela. La experiencia, vuelta un valor en sí misma, es recortada de sus relaciones estructurantes y se torna singularísima. El relevamiento por parte de las ciencias sociales de relatos experienciales en estas condiciones no solo ficcionaliza una individualidad solo creíble para el liberalismo político, sino que produce un "conocimiento" que carece de valor epistémico y político real. Los relatos biográficos presentan un valor para el conocimiento y para la política en tanto y en cuanto consiguen hablar de una historia, de un contexto, de relaciones sociales, etc.

Esta es la primera operación en la transmutación del sentido y del valor gnoseológico y político de la noción de experiencia, que supone un desplazamiento de la atención de las condiciones sociales que podrían develar los relatos hacia una atención recortada sobre el carácter egológico (es decir, organizado en función de la lógica del yo -ego-) de dichos relatos.

Dicha operación es acompañada, a su vez, por una segunda que supone la esencialización de la experiencia individual. Esta, ahora, se convierte en el recinto último de la verdad del sujeto. Se revierte de esta manera una oposición gnoseológica moderna que enfrentaba entendimiento y raciocinio, por un lado, a sensaciones y experiencias, por otro. Habiendo sido tradicionalmente las primeras las fuentes de la verdad, ahora son las experiencias y las sensaciones (ya recortadas de sus relaciones estructurantes) las que comportarían las llaves para la adquisición de una verdad de mayor grado ontológico sobre el sujeto. Los relatos experienciales se opondrían a los relatos desencarnados de la ciencia, sospechados de artificiosos $y$, por tanto, carentes de verdad. La experiencia se presenta como aquella que mejor habla la verdad última, esencial, del sujeto. Y hay que advertir aquí que verdad esencial no es igual a verdad. Es cierto que el relato experiencial es un relato que habla la verdad del sujeto, pero esa verdad no es ni natural ni esencial, es contingente, contextual y producida. 
La intensidad psicosomática con la que experimentamos algunas afecciones (el amor, la tristeza, el desengaño, el enfado, la ira, etc.) ha sido interpretada como evidencia de estar frente a experiencias verdaderas y esenciales del sujeto, en el sentido de naturales, en el sentido de no artificiales. La experiencia profunda del amor materno, por ejemplo, ha sido interpretado por muchísimo tiempo en esta clave. Acontece entonces una naturalización de las afecciones y una esencialización de la experiencia. Y ya Bourdieu se había ocupado de desmitificar lo natural en esas sensaciones que experimentamos como si provinieran desde nuestras fibras más íntimas y profundas.

Ellas, explica Bourdieu, son resultado de una buena (o mala) educación afectiva producida por los discursos performativos que sostienen y atraviesan todas las instituciones sociales por las que pasamos y en las que habitamos. Entonces, contra esta perspectiva que naturaliza nuestras afecciones, sentimientos y experiencias, hay que señalar que ellas no están al resguardo del artificio, y este no es el problema. Vivimos en los artificios, somos artificio. La trampa adviene cuando caemos presos/as de cierta ansiedad o pereza intelectual y (re)instalamos en algún lugar la ficción de la ausencia del artificio, del paraíso de la verdad última y no contaminada.

Esta es la segunda operación de la trasmutación del valor gnoseológico y político de la experiencia que supone la confusión de lo que es una verdad contingente, producida y coyuntural del sujeto con una supuesta verdad esencial del mismo. Solo la primera, por otra parte, es la que podría volverse camino para la contemplación de las condiciones estructurantes del sujeto, que son, nuevamente, las que aquí nos importan.

Llamo la atención sobre este punto porque advierto entre algunos de los (cada vez más números) estudios con perspectiva de género la aparición de un empleo de la experiencia cae, con más regularidad, en esta serie de desplazamientos y recortes de sentido. Y esta situación no es problemática porque le falta al procedimiento del contra-método, sino porque pierde de vista y olvida el carácter complejo e inapropiable de toda experiencia y es funcional a la reactualización de una matriz de inteligibilidad de las experiencias de las mujeres que discrimina qué experiencias son posibles de ser leídas como experiencias de mujeres y cuáles no. Sobre esta matriz de intelección trabajaré a continuación.

\section{ii. Institucionalización, demanda social y judicialización de los conflictos en la confección de una matriz de inteligibilidad de las experiencias}

El segundo asunto sobre el que quería llamar la atención refiere a la progresiva instalación de una matriz de inteligibilidad de las experiencias, resultado de una interpretación del funcionamiento de opresión de las mujeres, que anticipa las experiencias posibles de ser reconocidas como tales y las recorta de otras que no consiguen siquiera ser visualizadas. Tras la instalación de esta matriz se encuentran actuando: a) la legitimidad adquirida de los estudios feministas, b) la divulgación de los mismos (que conlleva la implementación de diversas políticas públicas) y c) el modo que nuestra sociedad encuentra disponible para dirimir los conflictos. Es cierto que esta matriz no se instala sin conflictos ni debates. Existen voces que, desde la calle y desde los textos, apuntan a conmover su rigidez. Sin embargo, su legitimidad para la fecha es incuestionable. Esta condición tan poco compatible tanto con el espíritu del conocimiento científico como con una política libertaria, debe empujarnos a una revisión. Concretamente a revisar la posible participación del discurso académico feminista en el refuerzo de dicha matriz endurecida.

Advertimos que la matriz de inteligibilidad de las experiencias a la que hacemos referencia resulta convenientemente coincidente con los términos a los que son necesariamente traducidos los conflictos 
sociales al momento de procurar una solución con los modos disponibles de la sociedad actual, es decir, vía su judicialización. En este punto, cabe, entonces, reparar en dos cuestiones: por un lado, en el modo en que se ve implicada la sensibilidad y el compromiso que las/os investigadoras/os con las demandas del movimiento de mujeres en el camino de la gestión de la justicia, que en ocasiones podría compeler a los/as investigadores/as a una construcción epistémicamente acrítica de testimonios o relatos experienciales, o meramente estratégica. $Y$, por otro lado, sería necesario analizar el modo en que el discurso de legal informado en teoría de género opera en la comprensión y la construcción de la propia experiencia de aquellos/as que son llamados/as a dar testimonio.

Los compromisos políticos que abraza la teoría feminista y la urgencia de un estado de cosas en el campo social que muestra un incremento en la violencia hacia las mujeres, podría quizás estar tensionando a los estudios a desalentar la exploración de las complejidades y de las contradicciones de los relatos vivenciales de los sujetos subalternos, prefiriendo quizás los lugares seguros de los diagnósticos ya aceptados por parte, por ejemplo, de instancias judiciales. Se produciría de este modo una suerte de yuxtaposición de los resultados de los estudios de género, la retórica de la demanda social y el discurso jurídico crítico informado en género.

Virginia Cano, en una conferencia titulada "Crítica de la razón femicida: sexualidad, amor y poder" (2016), ha analizado los avances innegables del ingreso de la denuncia feminista a la letra jurídica, pero también y siguiendo a Tammar Pich- ha sopesado los límites que impone el juego de la justicia punitiva en la resolución de conflictos. Un paradigma de gestión de los conflictos que, basado en la ficción del derecho liberal, resuelve problemas sociales en términos de responsabilidades individuales. Ahora además con una incorporación maniquea de la perspectiva del género que intenta subsanar vía la ley las injusticias sociales de la opresión sobre la población subalterna que son las mujeres, despliega estrategias de discriminación positiva, algunas de las cuales conllevan compromisos cuyos beneficios para las mujeres deben aún ser comprobados. El ensayo de una lengua del derecho informada en una perspectiva feminista ha venido enlazada a la asignación esencial de las mujeres al lugar de la debilidad y ausencia de poder. Los beneficios de esta discriminación positiva se pagan en otras instancias, pues se le ha sustraído a ella la posibilidad de la acción y de la violencia. En otras palabras, las experiencias que pueden ser leídas como "experiencias de las mujeres" para hacer andar la maquinaria de una justicia que ampare a las mujeres, tienen la necesidad de señalar, con exceso de linealidad, a estos sujetos como victimas pasivas. Y como contraparte necesaria de esta tendencia, quedan relegados aquellos otros relatos más confusos que hablan, por ejemplo, de estrategias y de negociaciones. Acciones éstas empoderantes que, sin embargo, descalificarían al sujeto a la hora de pedir justicia. Ahora bien, este requerimiento del discurso legal informado en teoría feminista no es el único problema ni el más importante.

Cano, siguiendo a Foucault, analiza al discurso jurídico como un "dispositivo de subjetivación, productor de los sujetos y de los saberes" (2016:2). De modo que, la verdad de los sujetos se presenta cristalizada en las formas jurídicas disponibles. Por su parte, Héctor Cárcamo (2016) ha señalado el modo en que la figuración del ciudadano (y todo el marco de significaciones asociadas) establece los límites para el modelamiento del sistema cognitivo en torno a la experiencia del sujeto. Este modelamiento de la figura del ciudadano (inscripta en las formas jurídicas es capitalista, liberal y heternormada) trabaja en torno a una subjetivación femenina de debilidad y a una subjetivación masculina de poder.

La eficacia del dispositivo jurídico de subjetivación puede ser puesta en entredicho, aunque solo de modo relativo, pues en tanto que las prácticas de militancia configuran sus problemas en términos de contienda legal, el mismo resulta reactualizado una y otra vez. El discurso jurídico se vuelve discurso performático 
por excelencia de la realidad social. De repente ya no es que traducimos nuestros problemas a sus términos, sino que sus términos definen nuestros problemas. Maquina productiva que, en lugar de quedar evidenciada por los estudios de investigación social, resulta, por el contrario, reforzada a partir de una consideración de los testimonios de las mujeres relevados en estudios sociales como expresiones indiscutidas de la verdad de las cosas (y no solo de la verdad contingente del sujeto). De este modo, la condición de víctima, por ejemplo, que debería ser pensada como una categoría jurídica o una estrategia desplegada por el sujeto del relato frente a una sociedad patriarcal, racista y capitalista que indudablemente depara lo peor para estos sujetos, termina siendo concebida como el relato que habla toda la verdad de la situación, obstruyendo de este modo la consideración de la complejidad de la realidad y de otras potencialidades de ese mismo sujeto.

Esta verdad esencial se sustenta en el olvido, por un lado, de la condición constructivista de todo relato de sí, que se estructura a partir de las categorías disponibles para narrarse y, por otro, de su condición dialógica, que funciona condicionando el relato experiencial en función del interlocutor especulado y las expectativas imaginadas de éste.

Es tarea de un feminismo crítico, epistémica y políticamente comprometido, revisar los lugares seguros a los que él mismo arribó. Sin dudas, la construcción de una matriz de inteligibilidad de las experiencias de los sujetos subalternos, antes imperceptibles, ha sido una victoria de la militancia y de la teoría feminista. Sin embargo, su institucionalización, vía legitimidad de la perspectiva, su divulgación social y la incorporación de políticas de estado, como en el discurso jurídico, ha conllevado algunos efectos indeseables que se expresan en la cerrazón en torno a las experiencias de las mujeres consideradas como posibles y en la cancelación de la diversidad agendas para el feminismo. No todas las feministas encuentran en la matriz de inteligibilidad de las experiencias disponible un organizador para leer nuestras realidades. Es de urgencia, por lo tanto, liberar las agendas del feminismo y para ello hay que tener disposición a desarmar la matriz, a que emerjan divergencias en las experiencias, en las lecturas y en los deseos de los sujetos.

\section{A modo de conclusión}

“...la encarnación feminista se opone a la fijación y es insaciablemente curiosa" (Haraway 1995:337).

La preocupación por la trasmutación del valor gnoseológico y político de la experiencia y por la instalación de una matriz de inteligibilidad de las experiencias (que han perdido ya su carácter de situado) debe interpelarnos porque ellas desandan el trabajoso camino que hemos construido desde la militancia y la teoría feminista. Ambas condiciones, asimismo, están de alguna manera ligadas al proceso de institucionalización académica y la consecuente legitimación social de la perspectiva feminista.

El feminismo siempre ha sido su propio, y mejor, crítico. Las feministas negras, latinas, lesbianas, etc., tempranamente le señalaron los límites a la agenda del feminismo blanco y heterosexual. La llamada interseccionalidad de la perspectiva feminista se consideró un triunfo del movimiento emancipatorio, pero quizás haya que dar otra vuelta, volver a desestabilizar los sentidos, ahora considerando la especificidad de la situación actual del feminismo académico, comprometido con un contexto social y político que en ocasiones delimita en exceso el pensamiento en función de lo posible, o más concretamente, de las disponibles vías de resolución de los conflictos. Debemos agudizar la vigilancia epistémica, revisar los compromisos adquiridos por la institucionalización y abandonarlos cuando sea necesario para que se abra la posibilidad a que emerjan otros diagnósticos sobre la opresión hacia los sujetos subalternos, pero 
también sobre la capacidad de resistencia y de subversión de los mismos, entre otras experiencias asociadas y otros (y nuevos) horizontes utópicos.

En este sentido el feminismo descolonial puede ser un camino en tanto y en cuanto alza su voz contrahegemónica para disputarle al feminismo hegemónico (institucionalizado, académico, estatal, pero también en ocasiones movimentista) las interpretaciones feministas de la opresión. En este sentido es un feminismo que revisa de modo radical el modo de resolución de los conflictos vía la ley y se propone pensar por fuera del dispositivo de subjetivación del discurso jurídico y ciudadano. Como señala Yudeskys Espinosa (2014), esta perspectiva apunta a producir un cambio epistemológico en el análisis de la opresión que conllevará necesariamente un cambio en el tipo de política a realizar y una transformación de los horizontes de utopía.

Rossi Braidotti (2004) en un texto en el que reflexionaba en torno a la progresiva adquisición de derechos de las mujeres blancas y europeas, expresaba un deseo. Decía que deseaba que las mujeres, esas mujeres, mantuvieran viva la memoria de lo que les costó estar dónde están ahora. En este mismo sentido, el texto histórico, epistémico y crítico que presento aquí quiere ser, finalmente, una propuesta para que mantengamos viva la memoria de cuánto nos costó estar donde estamos, aquí en la academia. Que sepamos recordar cuáles fueron los caminos epistémicos y políticos que elegimos tomar y por qué, con qué sentido. Que esta memoria nos lleve a recordar que los lugares demasiado seguros nunca fueron hospitalarios con nosotras, que desconfiemos de ellos, incluso si somos nosotras las que los (re)producimos. Que no abandonemos nunca las preguntas incómodas, la ironía, la sorpresa y la disidencia, en ninguna de sus formas. 


\section{Bibliografía}

Bourdieu, P; Chamboredon, J-C. y Passerron, J-C. 2008. El oficio del sociólogo. Presupuestos epistemológicos. Buenos Aires: Siglo XXI.

Braidotti, R. 2004. Feminismo, diferencia sexual y subjetividad nómade. Barcelona: Gedisa.

Cano, V. 2016. Crítica de la razón femicida: sexualidad, amor y poder. Conferencia dictada en el encuentro Sexualidades doctas: "resistir, disputar, coger". Córdoba (Argentina), 6 al 8 de abril.

Cárcamo, H. 2016. El sujeto como categoría de análisis de la ciudadanía. Cinta moebio 56: 231-242. doi: $10.4067 /$ S0717-554X2016000200009

Cornejo, R; Albornoz, N. y Palacios, D. 2016. Subjetividad, realidad y discurso entre el determinismo estructuralista y el construccionismo social. Cinta moebio 56: 121-135, doi: 10.4067/SO717$554 X 2016000200001$

Espinosa, Y. et. al. 2014. Tejiendo de otro modo: feminismo, epistemología y apuestas descoloniales en Abya Yala. Popayán: Editorial Universidad del Cauca.

Foucault, M. 1992. Microfísica del poder. Madrid: Ediciones La Piqueta.

Haraway, D. 1995. Ciencia, cyborgs y mujeres. La reinvención de la naturaleza. Madrid: Ediciones Cátedra.

Scott, J. W. 1992. Experience, pp. 22-40. En: J. Butler y J.W. Scott. Feminists theorize the political. New York: Routledge.

Smith, D. 2012. El punto de vista (standpoint) de las mujeres: Conocimiento encarnado versus relaciones de dominación. Temas de Mujeres 8(8): 5-27.

Recibido el 2 Sep 2016

Aceptado el 4 Nov 2016 